\title{
On the Publication of the Special Issue on Colloid and Interface
}

In general, a colloid is a heterogeneous mixture in which one phase or more is dispersed in the other phase, wherein the continuous phase is called 'medium' and the other phase is called 'dispersed phase'. Also, colloid is larger than molecule but small enough to maintain them dispersed under gravity (approximately less than $1 \mu \mathrm{m}$ ) due to their thermal energy. In a liquid medium, the dispersed phase can be solid, liquid or gas, which corresponds to (solid) sol, emulsion, or bubble, respectively. Similarly, a gas phase can also be a medium for solid or liquid dispersed phase, which is called solid or liquid aerosol. Along this line, interestingly, gas dispersed phase in solid matrix or 'porous materials' as well as solid nanoparticles in polymer matrix or 'polymer composite' are also classified as the colloid. Since such colloids are associated with enormously large interfacial area between two immiscible phases, understanding the related interfacial phenomena would be indispensable for the advanced colloid science and engineering.

Since colloids are heterogeneous mixtures, they are still new research areas that are not explored extensively yet in both fundamental science and engineering. Recently, along with discoveries or applications of new nanoscale phenomena, colloids at the comparable scale have taken lots of attention from both of scientists and engineers promoting significant progress in the colloidal science and engineering. This also accompanies a rapid and vast growth in related industries, such as chemical mechanical polishing (CMP), cleaning solutions in semiconductor fabrication, functional coatings in display, cosmetics, smart drug delivery in pharmaceutics, and high-performance electrodes for rechargeable batteries or solar cells.

In this special issue, there are four invited review articles on fast-growing state-of-the-art researches in colloid and interface, which includes DNA-coated colloids for programmable materials, transparent electrodes based on silver nanowires, liquid-crystal emulsions for structural colors and molecular cages for drug delivery, and fourteen communication or regular articles which are related to the synthesis of colloids, emulsions, biomedical, display or energy applications. Some of articles are contributed from invited speakers of the $2^{\text {nd }}$ Colloid and Interface Symposium (COINS 2018) which was held on June 4-5, 2018 in Sungkyunkwan University.

As Guest Editor for this special issue, I would like to thank all the authors for their excellent contributions and believe this issue will be of interest to all of our readers and well received by those in the colloid and interface community.

Gi-Ra Yi, Guest Editor Associate Professor, Chemical Engineering, Sungkyunkwan University

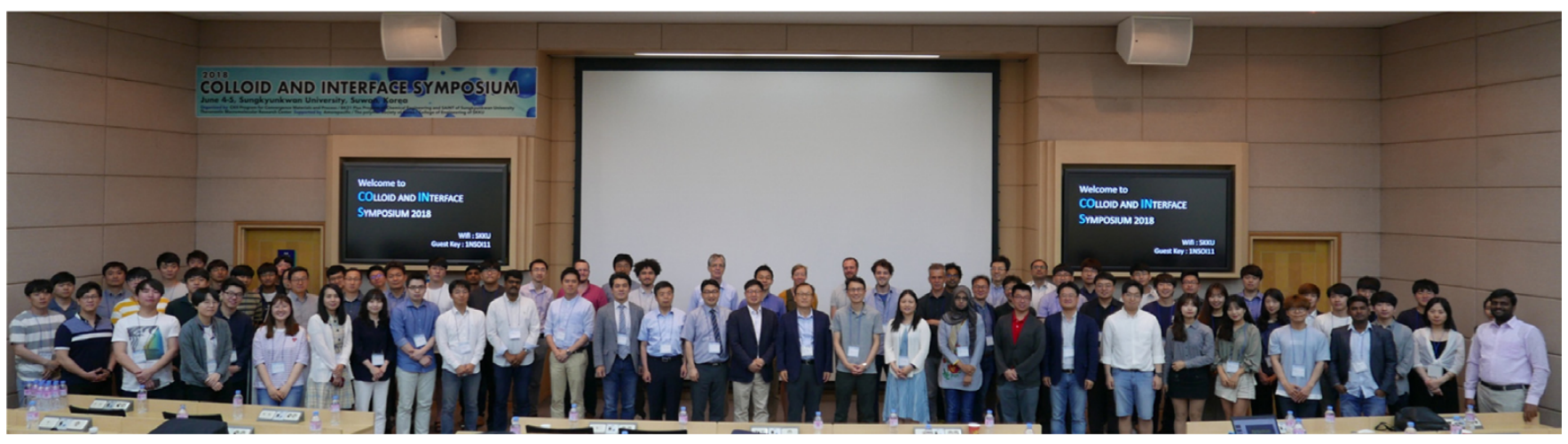

